$\sqrt{B}$

J. bio-sci. 22: 89-99, 2014

ISSN 1023-8654

http://www.banglajol.info/index.php/JBS/index

\title{
DEVELOPMENT OF ARTIFICIAL SEED AND PRESERVATION IN MIMOSA PUDICA L., AN IMPORTANT MEDICINAL PLANT IN BANGLADESH
}

\author{
L A Banu1', M Harun-Or-Rashid2², M A Bari Miah² \\ 1Upazilla Family Planning Office, Kalai, Jaypurhat, Bangladesh \\ ${ }^{2}$ Biotechnology Laboratory, Institute of Biological Sciences, University of Rajshahi-6205, Bangladesh
}

\begin{abstract}
Context: Mimosa pudica L. is an important medicinal plant belonging to the family- Mimosaceae has becoming a rare species in Bangladesh. The application of artificial seed technology using encapsulated shoot tips and nodal segments may contribute to the protection of rare and threatened medicinal plant like Mimosa pudica L.

Objective: Synthetic seed technology has been developed for Mimosa pudica L. in order to develop an alternative protocol on propagation and conservation.

Materials and Methods: For this purpose shoot tip and nodal segments obtained from in vitro grown plants were encapsulated with sodium alginate solution followed by subsequent immersion in $\mathrm{CaCl}_{2}$ solution. Different concentrations and combinations of growth regulators were used and explants were treated in alginate bead to investigate the hormonal effect on artificial seed germination. These encapsulated seeds were cultured either on MS medium with hormone (same growth regulators containing alginate beads) or $\mathrm{MS}_{0}$ (without hormone).

Results: Highest shoot regeneration frequency $(100 \%)$ were recorded when alginate beads were infused by MS medium supplemented with $2.0 \mathrm{mg} / \mathrm{l} \mathrm{BAP}+0.2 \mathrm{mg} / \mathrm{lNA}$ and cultured in MS medium containing same growth regulators. When synthetic seed containing $2.0 \mathrm{mg} / \mathrm{BAP}+0.2 \mathrm{mg} / \mathrm{l} \mathrm{NAA}$ and cultured on MSo medium, 54\% explants produced multifarious root with shoot in both cases. Under different storage period encapsulated seed retained germination capacity even after preserving for 60 days at $4^{\circ} \mathrm{C}$.

Conclusion: For artificial seed production a suitable protocol established under this study for Mimosa pudica L. that provides an alternative method for micropropagation and its conservation. For long term storage of Mimosa pudica in Bangladesh this protocol would provide promising avenues for the easy transference of propagules and its improvement.
\end{abstract}

Key words: Shoot tip, Nodal segments, Sodium alginate, Encapsulation, Micropropagation, Conservation.

\section{Introduction}

The artificial seed technology is an exciting and rapidly growing area of research in plant cell and tissue culture and unraveling new vistas in plant biotechnology. The idea of artificial seed was first conceived by Murashige et al. (1978) which was subsequently developed by several investigators. Initially, the development of artificial seeds has been restricted to encapsulation of somatic embryos in a protective jelly. It had been considered that the induction of somatic embryogenesis is the pre-requisite for the preparation of artificial seeds. But research on artificial seed has increased significantly, as demonstrated by increased attention from several laboratories (Kim and Janick 1987,Redembaugh et al. 1988, Gray 1987). The encapsulation technique is an important application of micropropagation that improves delivery of in vitro derived plants to the field or to greenhouse (Piccioni and Standardi 1995). In some crop species seed

* Corresponding author Email:harun.ibscru@gmail.com 
propagation has not been successful mainly due to heterozygosity, minute seed size, presence of reduced endosperm and the requirement of seed with mycorrhizal fungi association for germination (eg. orchids), and also in some seedless crop plants like grapes and watermelon. Development of artificial seed production technology is currently considered as an effective and efficient alternate method of propagation in several commercially important agronomic and horticultural crops.

Naturally most of the important medicinal plants are rare, endangered and endemic category. It is due to the low fruit and seed formation, poor germination capacity of seeds and due to the many other environmental conditions such as habitat modification, urbanization, climatic change and pollution etc. So, it is important to propagate and conserve these plant species. The production of synthetic seeds by encapsulating somatic embryos and vegetative propagules is rapidly becoming an applied technique with potential for mass propagation of medicinal plant species. Propagation of Valeriana wallichii using encapsulated apical and axial shoot buds with plantlet conversion under both in vitro (98\%) and in vivo (64\%) was reported by Mathur et al. (1989). Hasan and Takagi (1995) used nodal segments for encapsulation in Dioscorea spp. Alginate encapsulations of axillary buds of Ocimum sanctum, Ocimum basilicum, Ocimum americanum, Ocimum gratissimum and their growth even after 60 days of storage at $4^{\circ} \mathrm{C}$ were reported by Mandal et al. (2002). Pandey and Chand (2005) reported that the efficient plant regeneration (60\%) from encapsulated somatic embryos of Hyoscyamus muticus. Synthetic seeds were produced using in vitro proliferated shoots of Rauvolfia serpentina when $3 \%$ sodium alginate prepared in Llyod and McCown woody plant medium (WPM) and $100 \mathrm{mM}$ calcium chloride (Faisal et al. 2012 ).

In tissue culture, Mimosa pudica produced profuse multiple shoots and providing abundant nodal segments for the production of artificial seeds by encapsulating with sodium alginate. Mimosa pudica is rather an ideal plant species for establishing this protocol of artificial seed production in medicinal plants in Bangladesh. So far we know, no studies concerning artificial seed development and preservation in Mimosa pudica L. have yet been reported. Under the present investigation in vitro grown plants with abundant shoot tips and nodal segments were used for encapsulation of artificial seed for Mimosa pudica as an important medicinal plants in Bangladesh.

\section{Materials and Methods}

Shoot tip and nodal segments of Mimosa pudica L. were used as explants for artificial seed production. Shoot tips and nodal segments 3-4 mm long were aseptically excised from in vitro grown plants following the method of Maruyama et. al. (1996).

\section{A flow chart for production of artificial seeds of Mimosa pudica $L$}

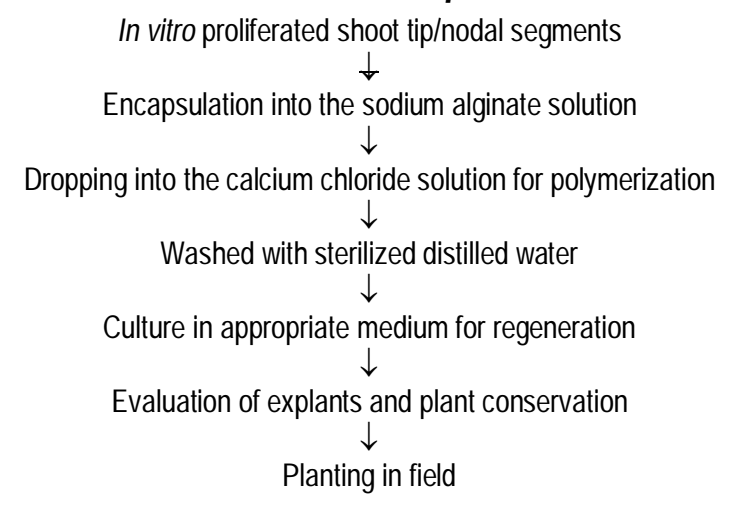

Under this study MS (Murashige and Skoog 1962) medium with $8 \mathrm{~g} / \mathrm{l}$ agar used. Sodium alginate beads were produced by encapsulation according to the method of Kinoshita and Satio (1990). MS medium (200 ml) was 
prepared with $6 \mathrm{gm}$ sucrose. $20 \mathrm{ml}$ MS medium taken in a small beaker $(50 \mathrm{ml})$ and $0.8 \mathrm{gm}$ of sodium alginate added with required growth regulator. A small piece of glass rod used to mix the alginate in solution. Alginate was partially dissolved and it was then kept aside. During autoclaving alginate was completely dissolved. In another lot, $50 \mathrm{ml} \mathrm{MS}$ medium taken in a small beaker and $0.7 \mathrm{gm} \mathrm{CaCl}$ was added and dissolved. Out of $200 \mathrm{ml} \mathrm{MS}$ medium, $70 \mathrm{ml}$ was used during the preparation of alginate and $\mathrm{CaCl}_{2}$ solution. Remaining $130 \mathrm{ml}$ used for washing the encapsulated beads.

The nodal segments with viable buds and shoot tips were placed to the beaker containing alginate solution. The explants were dipped in alginate solution. Then explants were taken by a forcep and placed to the beaker of $\mathrm{CaCl}_{2}$. After 30 minutes each explants become a hardball encoated by alginate. The encapsulated explants or artificial seeds were washed with $130 \mathrm{ml}$ MS liquid medium. After washing the artificial seeds were cultured in growth medium similarly supplemented with and without growth regulators and tested for preservation duration.

After developing sufficient root system and healthy growth the regenerated plantlets from artificial seed were brought out of the controlled environment of growth chamber and were kept in the room temperature for 3-4 days to bring them in contact to normal temperature. Well rooted plants taken out from the culture tubes and very carefully washed under running tap water for complete removal of remains of the medium. Then the plantlets were transplanted to small pots containing garden soil.

The soil was treated with $0.1 \%$ formalin solution. After transplantation the plants along with pots were covered with moist polythene bag to prevent desiccation. The interior portion of the polythene bags was sprayed with water every day to maintain high humidity around the plants. The polythene bags were completely removed after 5-7 days. When the plantlets grew well, they were transferred to garden.

\section{Results}

In artificial seed, explants were encoated with sodium alginate accompanied by required growth regulator which together acts like endosperm providing nutrients for germination of artificial seed. Under the present investigation experiments were conducted to test the effect of growth hormone applied in embedded seed coat in artificial seed also for studying its preservation duration.

\section{Seed capsule without hormone:}

This experiment was conducted to investigate the effect of $\mathrm{MS}_{0}$ for seed germination. For this purpose artificial seed was prepared without growth regulators in MS medium and similarly cultured on MS medium without hormone. Observation was made for 28 days but no germination of artificial seed was observer on MS 0 medium. The experiment proved that without growth regulator artificial seed embedded in capsulated bead fail to germinate.

\section{Seed capsule with hormone:}

Different concentrations of cytokinins (BAP and Kin) were used singly or in combinations with auxins (NAA, IAA and IBA) in seed bead to investigate the germination rate of artificial seeds and its subsequent growth. Encapsulated synthetic seeds were cultured on MSo medium and or MS medium containing same growth regulators as encapsulated in the synthetic seeds. Data on days of germination, percentage of seed germination, average number of shoot and average length of shoots were recorded after 28 days of culture and the results are briefly described below

\section{Effect of BAP on artificial seed production}

The encapsulated synthetic seeds impregnated with different concentrations of BAP (1.0, 2.0 and $3.0 \mathrm{mg} / \mathrm{l})$ were cultured either on MS medium with hormone (same growth regulators) or $\mathrm{MS}_{0}$ (without hormone). Results of this experiment are presented in Table 1. Best rate of germination (94\%) was observed when 2.0 $\mathrm{mg} / \mathrm{BAP}$ was used in synthetic seed containing same growth regulators as used in regeneration medium. 
The germination rate was $94 \%$ for shoot tip and $87 \%$ for nodal segments. In this concentration, days to germination were 5-7 for shoot tip and 7-8 for nodal explant. Maximum number of shoot and its length was observed in this concentration after 28 days. Average shoot number was $19.43 \pm 0.90$ for shoot tip explants and $14.38 \pm 0.71$ for nodal explants. On the other hand shoot length was $3.36 \pm 0.83 \mathrm{~cm}$ for shoot tip and $3.05 \pm 0.54 \mathrm{~cm}$ for nodal explants respectively.

When synthetic seed containing $2.0 \mathrm{mg} / \mathrm{l} \mathrm{BAP}$ and they cultured on MSo medium, the frequency of germination was $80 \%$ for shoot tip and $74 \%$ in nodal explants. In this treatment germination period was $8-10$ days for shoot tip and 10-12 days for nodal explants. But when synthetic seed containing $3.0 \mathrm{mg} / \mathrm{BAP}$ cultured on MS medium, the germination rate was decreased for both explants. Here $60 \%$ synthetic seed for shoot tip and $67 \%$ seed for nodal explants were germinated and time period varied 10-14 days for shoot tip and 12-15 days for nodal explants.

\section{Effect of Kin on synthetic seed production}

Different concentrations of Kin $(1.0,2.0$ and $3.0 \mathrm{mg} / \mathrm{l})$ were investigated on artificial seed. Similarly, encapsulated synthetic seeds were cultured on MS medium containing same growth regulators or MSo medium (Table 1). In this experiment, highest percentage of seed germination (54\%) was observed when 2.0 $\mathrm{mg} / \mathrm{l} \mathrm{Kin} \mathrm{was} \mathrm{used} \mathrm{in} \mathrm{synthetic} \mathrm{seed} \mathrm{with} \mathrm{same} \mathrm{growth} \mathrm{regulator} \mathrm{in} \mathrm{MS} \mathrm{regeneration} \mathrm{medium.} \mathrm{In} \mathrm{this} \mathrm{case}$ $54 \%$ seed of shoot tip and $47 \%$ seed of nodal explants were germinated and seeds were germinated after 16-18 days of culture in shoot tip and after 17-18 days of culture in nodal explants. When synthetic seed containing $3.0 \mathrm{mg} / \mathrm{l}$ Kin were cultured on MSo medium, the germination rate was lowest for both explants. In this case $27 \%$ synthetic seed were germinated in shoot tip and $20 \%$ synthetic seed were germinated in nodal explants. Seed were germinated after 20-24 days of culture in both explants. Synthetic seed containing 1.0 $\mathrm{mg} / \mathrm{l} \mathrm{Kin} \mathrm{cultured} \mathrm{on} \mathrm{MS}_{0}$ medium, the explants produced root with shoot and $34 \%$ synthetic seed induced root in both explants.

\section{Effect of BAP with NAA on artificial seed production:}

Germination was observed when the alginate beads were infused with MS medium supplemented with 1.0 $\mathrm{mg} / \mathrm{l} \mathrm{BAP}$ and $2.0 \mathrm{mg} / \mathrm{l} \mathrm{BAP}$ and three concentrations $(0.1,0.2$ and $0.5 \mathrm{mg} / \mathrm{l})$ of NAA. For comparative analysis these synthetic seed were cultured on MS medium containing same growth regulators and MS medium without any growth regulators (Table 2).

When shoot tip and nodal segments were used as explants for synthetic seed production $0.1,0.2$ and 0.5 $\mathrm{mg} / \mathrm{l} \mathrm{NAA}$ with $2.0 \mathrm{mg} / \mathrm{BAP}$ enhanced the germination rate. Synthetic seed supplemented with $2.0 \mathrm{mg} / \mathrm{l} \mathrm{BAP}$ $+0.2 \mathrm{mg} / \mathrm{l} \mathrm{NAA}$ and cultured on MS medium containing same growth regulators was proved to be the best medium composition for better germination $(100 \%)$ for artificial seed. In this concentration duration for germination was 3-5 days for shoot tip and 4-5 days for nodal explants. Maximum number and length of shoot was observed in this combination. Average shoot number was $14.93 \pm 0.64$ for shoot tip explants and $14.53 \pm 0.54$ for nodal explants and shoot length was $9.47 \pm 0.96 \mathrm{~cm}$ for shoot tip and $8.33 \pm 0.15 \mathrm{~cm}$ for nodal explants respectively. Cent percent seed germination was also observed in $2.0 \mathrm{mg} / \mathrm{l} \mathrm{BAP}+0.5 \mathrm{mg} / \mathrm{l} \mathrm{NAA}$ and $2.0 \mathrm{mg} / \mathrm{l} \mathrm{BAP}+0.1 \mathrm{mg} / \mathrm{l} \mathrm{NAA}$.

When synthetic seed containing $2.0 \mathrm{mg} / \mathrm{BAP}+0.2 \mathrm{mg} / \mathrm{l} \mathrm{NAA}$ cultured on $\mathrm{MS}_{0}$ medium, the frequency of germination was $87 \%$ in shoot tip and $80 \%$ in nodal explants. Duration of germination was 5-6 days in both explants. In this concentration $54 \%$ explant produced root with shoot in both cases. On the other hand, synthetic seed containing $1.0 \mathrm{mg} / \mathrm{BAP}+0.1 \mathrm{mg} / \mathrm{lNAA}$ cultured on $\mathrm{MS}_{0}$ medium, the germination rate and number of shoot was lowest for both explants. Here, $67 \%$ synthetic seed were germinated and duration of germination was 7-10 days in both explants. 
Table 1. Effect of MSo and different concentrations of BAP and Kin on synthetic seed production.

\begin{tabular}{|c|c|c|c|c|c|c|c|}
\hline \multicolumn{2}{|c|}{ Growth regulators in } & \multirow[b]{2}{*}{ Explants } & \multirow{2}{*}{$\begin{array}{c}\text { Days of } \\
\text { germination }\end{array}$} & \multirow{2}{*}{$\begin{array}{c}\% \text { of } \\
\text { germination }\end{array}$} & \multirow[b]{2}{*}{$\begin{array}{c}\text { Average number of } \\
\text { shoots } \pm S \text {. } E \text {. }\end{array}$} & \multirow{2}{*}{$\begin{array}{l}\text { Average length } \\
(\mathrm{cm}) \text { of shoots } \\
\pm \mathrm{S} \text {. E. }\end{array}$} & \multirow[b]{2}{*}{$\begin{array}{l}\% \text { of root } \\
\text { induction }\end{array}$} \\
\hline $\begin{array}{l}\text { Culture } \\
\text { medium }\end{array}$ & $\begin{array}{l}\text { Alginate } \\
\text { beads }\end{array}$ & & & & & & \\
\hline \multirow{2}{*}{$\mathrm{MS}_{0}$} & \multirow{2}{*}{$\mathrm{MS}_{0}$} & Shoot tip & - & - & - & - & - \\
\hline & & Node & - & - & - & - & - \\
\hline \multirow{2}{*}{$\mathrm{MS}_{0}$} & \multirow{2}{*}{$\begin{array}{c}1.0 \mathrm{mg} / \mathrm{l} \\
\mathrm{BAP}\end{array}$} & Shoot tip & $8-12$ & 74 & $6.27 \pm 0.13$ & $2.05 \pm 0.94$ & - \\
\hline & & Node & $10-12$ & 67 & $4.80 \pm 0.94$ & $1.86 \pm 0.41$ & - \\
\hline \multirow{2}{*}{$\mathrm{MS}_{0}$} & \multirow{2}{*}{$\begin{array}{c}1.0 \mathrm{mg} / \mathrm{l} \\
\mathrm{Kin}\end{array}$} & Shoot tip & $17-20$ & 42 & $1.00 \pm 0.00$ & $0.70 \pm 0.18$ & 34 \\
\hline & & Node & $17-20$ & 27 & $1.00 \pm 0.00$ & $0.63 \pm 0.17$ & 34 \\
\hline \multirow{2}{*}{$\begin{array}{c}1.0 \mathrm{mg} / \mathrm{l} \\
\text { BAP }\end{array}$} & \multirow{2}{*}{$\begin{array}{c}1.0 \mathrm{mg} / \mathrm{l} \\
\text { BAP }\end{array}$} & Shoot tip & $6-8$ & 80 & $15.75 \pm 0.88$ & $3.10 \pm 0.87$ & - \\
\hline & & Node & $6-8$ & 87 & $13.85 \pm 0.59$ & $2.15 \pm 0.72$ & - \\
\hline \multirow{2}{*}{$\begin{array}{c}1.0 \mathrm{mg} / \mathrm{l} \\
\text { Kin }\end{array}$} & \multirow{2}{*}{$\begin{array}{c}1.0 \mathrm{mg} / \mathrm{l} \\
\text { Kin }\end{array}$} & Shoot tip & 15- 17 & 47 & $1.00 \pm 0.00$ & $1.29 \pm 0.37$ & - \\
\hline & & Node & $15-17$ & 40 & $1.00 \pm 0.00$ & $0.87 \pm 0.25$ & - \\
\hline \multirow{2}{*}{$\mathrm{MS}_{0}$} & \multirow{2}{*}{$\begin{array}{l}2.0 \mathrm{mg} / \mathrm{l} \\
\text { BAP }\end{array}$} & Shoot tip & $8-10$ & 80 & $5.17 \pm 0.83$ & $3.00 \pm 0.65$ & 47 \\
\hline & & Node & $10-12$ & 74 & $5.27 \pm 0.80$ & $1.94 \pm 0.43$ & 47 \\
\hline \multirow{2}{*}{$\mathrm{MS}_{0}$} & \multirow{2}{*}{$\begin{array}{l}2.0 \mathrm{mg} / \mathrm{l} \\
\mathrm{Kin}\end{array}$} & Shoot tip & $16-20$ & 34 & $1.00 \pm 0.00$ & $0.82 \pm 0.37$ & - \\
\hline & & Node & $18-20$ & 34 & $1.00 \pm 0.00$ & $0.84 \pm 0.36$ & - \\
\hline \multirow{2}{*}{$\begin{array}{c}2.0 \mathrm{mg} / \mathrm{l} \\
\text { BAP }\end{array}$} & \multirow{2}{*}{$\begin{array}{l}2.0 \mathrm{mg} / \mathrm{l} \\
\text { BAP }\end{array}$} & Shoot tip & $5-7$ & 94 & $19.43 \pm 0.90$ & $3.36 \pm 0.83$ & - \\
\hline & & Node & $7-8$ & 87 & $14.38 \pm 0.71$ & $3.05 \pm 0.54$ & - \\
\hline \multirow{2}{*}{$\begin{array}{c}2.0 \mathrm{mg} / \mathrm{l} \\
\mathrm{Kin}\end{array}$} & \multirow{2}{*}{$\begin{array}{c}2.0 \mathrm{mg} / \mathrm{l} \\
\mathrm{Kin}\end{array}$} & Shoot tip & $16-18$ & 54 & $1.13 \pm 0.19$ & $1.20 \pm 0.47$ & - \\
\hline & & Node & $17-18$ & 47 & $1.14 \pm 0.13$ & $0.87 \pm 0.30$ & - \\
\hline \multirow{2}{*}{ MSo } & \multirow{2}{*}{$\begin{array}{l}3.0 \mathrm{mg} / \mathrm{l} \\
\text { BAP }\end{array}$} & Shoot tip & $10-14$ & 60 & $4.50 \pm 0.57$ & $2.44 \pm 0.60$ & - \\
\hline & & Node & $12-14$ & 67 & $4.22 \pm 0.62$ & $1.64 \pm 0.38$ & - \\
\hline \multirow{2}{*}{$\mathrm{MS}_{0}$} & \multirow{2}{*}{$\begin{array}{c}3.0 \mathrm{mg} / \mathrm{l} \\
\mathrm{Kin}\end{array}$} & Shoot tip & $20-24$ & 27 & $1.00 \pm 0.00$ & $0.70 \pm 0.13$ & - \\
\hline & & Node & $20-24$ & 20 & $1.00 \pm 0.00$ & $0.63 \pm 0.15$ & - \\
\hline \multirow{2}{*}{$\begin{array}{l}3.0 \mathrm{mg} / \mathrm{l} \\
\text { BAP }\end{array}$} & \multirow{2}{*}{$\begin{array}{l}3.0 \mathrm{mg} / \mathrm{l} \\
\text { BAP }\end{array}$} & Shoot tip & $6-10$ & 87 & $13.46 \pm 0.70$ & $2.63 \pm 0.37$ & - \\
\hline & & Node & $8-12$ & 80 & $10.92 \pm 0.91$ & $2.52 \pm 0.59$ & - \\
\hline $3.0 \mathrm{mg} / \mathrm{l}$ & $3.0 \mathrm{mg} / \mathrm{l}$ & Shoot tip & $18-22$ & 40 & $1.00 \pm 0.00$ & $0.95 \pm 0.57$ & - \\
\hline Kin & Kin & Node & $17-22$ & 34 & $1.00 \pm 0.00$ & $1.00 \pm 0.33$ & - \\
\hline
\end{tabular}


Table 2. Effect of MSo and different concentrations of BAP combination with NAA on synthetic seed production.

\begin{tabular}{|c|c|c|c|c|c|c|c|}
\hline \multicolumn{2}{|c|}{ Growth regulators in } & \multirow[b]{2}{*}{ Explants } & \multirow{2}{*}{$\begin{array}{c}\text { Days of } \\
\text { germination }\end{array}$} & \multirow{2}{*}{$\begin{array}{c}\% \text { of } \\
\text { germination }\end{array}$} & \multirow{2}{*}{$\begin{array}{l}\text { Average number of } \\
\text { shoots } \pm S . E \text {. }\end{array}$} & \multirow{2}{*}{$\begin{array}{l}\text { Average length }(\mathrm{cm}) \\
\text { of shoots } \pm S . E .\end{array}$} & \multirow{2}{*}{$\begin{array}{l}\% \text { of root } \\
\text { induction }\end{array}$} \\
\hline $\begin{array}{l}\text { Culture } \\
\text { medium }\end{array}$ & $\begin{array}{c}\text { Alginate } \\
\text { beads }\end{array}$ & & & & & & \\
\hline \multirow[b]{2}{*}{$\mathrm{MS}_{0}$} & \multirow{2}{*}{$\begin{array}{c}1.0 \mathrm{mg} / \mathrm{l} \mathrm{BAP} \\
+0.1 \mathrm{mg} / \mathrm{l} \\
\text { NAA }\end{array}$} & Shoot tip & $7-10$ & 67 & $6.60 \pm 0.78$ & $5.20 \pm 0.70$ & - \\
\hline & & Node & $7-10$ & 67 & $5.90 \pm 0.04$ & $4.90 \pm 0.69$ & - \\
\hline \multirow{2}{*}{$\begin{array}{c}1.0 \mathrm{mg} / \mathrm{l} \mathrm{BAP} \\
+0.1 \mathrm{mg} / \mathrm{l} \\
\text { NAA }\end{array}$} & \multirow{2}{*}{$\begin{array}{c}1.0 \mathrm{mg} / \mathrm{l} \mathrm{BAP} \\
+0.1 \mathrm{mg} / \mathrm{l} \\
\text { NAA }\end{array}$} & Shoot tip & $6-8$ & 80 & $7.58 \pm 0.15$ & $5.50 \pm 0.43$ & - \\
\hline & & Node & $7-8$ & 74 & $6.45 \pm 0.18$ & $5.45 \pm 0.35$ & - \\
\hline \multirow{2}{*}{$\mathrm{MS}_{0}$} & \multirow{2}{*}{$\begin{array}{c}1.0 \mathrm{mg} / \mathrm{l} \mathrm{BAP} \\
+0.2 \mathrm{mg} / \mathrm{l} \\
\text { NAA }\end{array}$} & Shoot tip & $6-8$ & 80 & $7.75 \pm 0.52$ & $5.33 \pm 0.90$ & - \\
\hline & & Node & $7-8$ & 74 & $7.55 \pm 0.75$ & $5.18 \pm 0.66$ & - \\
\hline \multirow{2}{*}{$\begin{array}{c}1.0 \mathrm{mg} / \mathrm{l} \mathrm{BAP} \\
+0.2 \mathrm{mg} / \mathrm{l} \\
\mathrm{NAA}\end{array}$} & \multirow{2}{*}{$\begin{array}{c}1.0 \mathrm{mg} / \mathrm{l} \mathrm{BAP} \\
+0.2 \mathrm{mg} / \mathrm{l} \\
\text { NAA }\end{array}$} & Shoot tip & $5-6$ & 87 & $10.77 \pm 0.59$ & $5.85 \pm 0.93$ & - \\
\hline & & Node & $5-7$ & 87 & $9.00 \pm 0.52$ & $5.62 \pm 0.92$ & - \\
\hline \multirow{2}{*}{$\mathrm{MS}_{0}$} & \multirow{2}{*}{$\begin{array}{c}1.0 \mathrm{mg} / \mathrm{l} \mathrm{BAP} \\
+0.5 \mathrm{mg} / \mathrm{l} \\
\text { NAA }\end{array}$} & Shoot tip & $7-9$ & 74 & $7.36 \pm 0.80$ & $5.09 \pm 0.48$ & - \\
\hline & & Node & $7-9$ & 67 & $6.20 \pm 0.93$ & $5.00 \pm 0.67$ & - \\
\hline \multirow{2}{*}{$\begin{array}{c}1.0 \mathrm{mg} / \mathrm{l} \mathrm{BAP} \\
+0.5 \mathrm{mg} / \mathrm{l} \\
\text { NAA }\end{array}$} & \multirow{2}{*}{$\begin{array}{c}1.0 \mathrm{mg} / \mathrm{l} \mathrm{BAP} \\
+0.5 \mathrm{mg} / \mathrm{l} \\
\mathrm{NAA}\end{array}$} & Shoot tip & $6-7$ & 87 & $7.77 \pm 0.91$ & $5.69 \pm 0.83$ & - \\
\hline & & Node & $6-7$ & 80 & $7.33 \pm 0.17$ & $5.50 \pm 0.94$ & - \\
\hline \multirow[b]{2}{*}{ MSo } & \multirow{2}{*}{$\begin{array}{c}2.0 \mathrm{mg} / \mathrm{l} \mathrm{BAP} \\
+0.1 \mathrm{mg} / \mathrm{l} \\
\text { NAA }\end{array}$} & Shoot tip & $5-7$ & 87 & $7.76 \pm 0.68$ & $4.69 \pm 0.91$ & - \\
\hline & & Node & $5-7$ & 80 & $7.25 \pm 0.96$ & $4.29 \pm 0.95$ & - \\
\hline \multirow{2}{*}{$\begin{array}{c}2.0 \mathrm{mg} / \mathrm{l} \mathrm{BAP} \\
+0.1 \mathrm{mg} / \mathrm{l} \\
\mathrm{NAA}\end{array}$} & \multirow{2}{*}{$\begin{array}{c}2.0 \mathrm{mg} / \mathrm{l} \mathrm{BAP} \\
+0.1 \mathrm{mg} / \mathrm{l} \\
\mathrm{NAA}\end{array}$} & Shoot tip & $4-6$ & 100 & $13.93 \pm 0.18$ & $8.07 \pm 0.89$ & - \\
\hline & & Node & $4-6$ & 94 & $12.29 \pm 0.43$ & $7.86 \pm 0.44$ & - \\
\hline \multirow[b]{2}{*}{$\mathrm{MS}_{0}$} & \multirow{2}{*}{$\begin{array}{c}2.0 \mathrm{mg} / \mathrm{l} \mathrm{BAP} \\
+0.2 \mathrm{mg} / \mathrm{l} \\
\text { NAA }\end{array}$} & Shoot tip & $5-6$ & 87 & $8.00 \pm 0.88$ & $4.91 \pm 0.82$ & 54 \\
\hline & & Node & $5-6$ & 80 & $6.67 \pm 0.77$ & $4.80 \pm 0.90$ & 54 \\
\hline \multirow{2}{*}{$\begin{array}{c}2.0 \mathrm{mg} / \mathrm{l} \mathrm{BAP} \\
+0.2 \mathrm{mg} / \mathrm{l} \\
\text { NAA }\end{array}$} & \multirow{2}{*}{$\begin{array}{c}2.0 \mathrm{mg} / \mathrm{l} \mathrm{BAP} \\
+0.2 \mathrm{mg} / \mathrm{l} \\
\text { NAA }\end{array}$} & Shoot tip & $3-5$ & 100 & $14.93 \pm 0.64$ & $9.47 \pm 0.96$ & - \\
\hline & & Node & 4- 5 & 100 & $14.53 \pm 0.54$ & $8.33 \pm 0.15$ & - \\
\hline \multirow{2}{*}{$\mathrm{MS}_{0}$} & \multirow{2}{*}{$\begin{array}{c}2.0 \mathrm{mg} / \mathrm{l} \mathrm{BAP} \\
+0.5 \mathrm{mg} / \mathrm{l} \\
\text { NAA }\end{array}$} & Shoot tip & $5-9$ & 80 & $7.08 \pm 0.19$ & $4.78 \pm 0.44$ & 54 \\
\hline & & Node & $5-9$ & 80 & $6.42 \pm 0.90$ & $4.35 \pm 0.31$ & 54 \\
\hline \multirow{2}{*}{$\begin{array}{c}2.0 \mathrm{mg} / \mathrm{l} \mathrm{BAP} \\
+0.5 \mathrm{mg} / \mathrm{l} \\
\mathrm{NAA} \\
\end{array}$} & \multirow{2}{*}{$\begin{array}{c}2.0 \mathrm{mg} / \mathrm{l} \mathrm{BAP} \\
+0.5 \mathrm{mg} / \mathrm{l} \\
\mathrm{NAA}\end{array}$} & Shoot tip & $5-6$ & 100 & $11.60 \pm 0.84$ & $6.07 \pm 0.59$ & - \\
\hline & & Node & $5-6$ & 100 & $11.20 \pm 0.78$ & $5.45 \pm 0.90$ & - \\
\hline
\end{tabular}

\section{Effect of $B A P+I B A$ on artificial seed production}

Three different concentrations of IBA $(0.1,0.2$ and $0.5 \mathrm{mg} / \mathrm{l})$ were also used in the combination with BAP and the results are presented in Table 3 . Best result was observed when synthetic seed and regeneration medium (MS) both containing growth regulators $2.0 \mathrm{mg} / \mathrm{l} \mathrm{BAP}+0.1 \mathrm{mg} / \mathrm{l} \mathrm{IBA}$. In this concentration $67 \%$ seed for shoot tip explants was germinated within 30-35 days of culture and $60 \%$ seeds for nodal explants within 32-38 days of culture. Maximum average number of shoot was obtained in synthetic seed and media 
containing $1.0 \mathrm{mg} / \mathrm{lBAP}+0.2 \mathrm{mg} / \mathrm{IBA}$. In this concentration $1.71 \pm 0.17$ number of shoot was obtained from shoot tip and $1.43 \pm 0.18$ number of shoot was obtained from nodal explants after 35-38 days of culture. But when synthetic seed containing $2.0 \mathrm{mg} / \mathrm{l} \mathrm{BAP}+0.1 \mathrm{mg} / \mathrm{l}$ IBA cultured on MS medium without growth regulators, $47 \%$ of seed for shoot tip explant and $40 \%$ of seed for nodal explant was germinated after 38-40 days of culture. The average number of shoot was calculated $1.43 \pm 0.27$ in shoot tip and $1.34 \pm 0.19$ in nodal explants. Lowest result was observed in synthetic seed containing $2.0 \mathrm{mg} / \mathrm{BAP}+0.5 \mathrm{mg} / \mathrm{l}$ IBA cultured on MS medium without growth regulators. Under this study all the tested concentrations germinating artificial roots found to induce roots.

Table 3. Effect of MS 0 and different concentrations of BAP combination with IBA on synthetic seed production.

\begin{tabular}{|c|c|c|c|c|c|c|c|}
\hline \multicolumn{2}{|c|}{ Growth regulators in } & \multirow[b]{2}{*}{ Explants } & \multirow{2}{*}{$\begin{array}{l}\text { Days of } \\
\text { germination }\end{array}$} & \multirow{2}{*}{$\begin{array}{c}\% \text { of } \\
\text { germination }\end{array}$} & \multirow{2}{*}{$\begin{array}{c}\text { Average } \\
\text { number of } \\
\text { shoots } \pm S . E .\end{array}$} & \multirow{2}{*}{$\begin{array}{l}\text { Average length }(\mathrm{cm}) \\
\text { of shoots } \pm S . E .\end{array}$} & \multirow{2}{*}{$\begin{array}{l}\% \text { of roo } \\
\text { induction }\end{array}$} \\
\hline $\begin{array}{l}\text { Culture } \\
\text { medium }\end{array}$ & $\begin{array}{l}\text { Alginate } \\
\text { beads }\end{array}$ & & & & & & \\
\hline \multirow{2}{*}{$\mathrm{MS}_{0}$} & 1.0 mg/l BAP+ & Shoot tip & $40-42$ & 27 & $1.50 \pm 0.25$ & $1.00 \pm 00$ & 27 \\
\hline & $0.1 \mathrm{mg} / \mathrm{l} \mathrm{IBA}$ & Node & $40-45$ & 20 & $1.33 \pm 0.27$ & $1.00 \pm 0.23$ & 20 \\
\hline \multicolumn{2}{|c|}{$1.0 \mathrm{mg} / \mathrm{BAP}+1.0 \mathrm{mg} / \mathrm{l} \mathrm{BAP}+$} & Shoot tip & $35-40$ & 40 & $1.67 \pm 0.19$ & $1.00 \pm 0.20$ & 40 \\
\hline 0.1 mg/l IBA & 0.1 mg/l IBA & Node & $38-40$ & 34 & $1.40 \pm 0.21$ & $1.00 \pm 0.24$ & 34 \\
\hline \multirow{2}{*}{ MSo } & $1.0 \mathrm{mg} / \mathrm{lBAP}+$ & Shoot tip & $40-45$ & 34 & $1.40 \pm 0.21$ & $1.20 \pm 0.22$ & 34 \\
\hline & $0.2 \mathrm{mg} / \mathrm{lBA}$ & Node & $40-45$ & 20 & $1.33 \pm 0.27$ & $1.16 \pm 0.36$ & 20 \\
\hline \multicolumn{2}{|c|}{$1.0 \mathrm{mg} / \mathrm{l} \mathrm{BAP}+1.0 \mathrm{mg} / \mathrm{BAP}+$} & Shoot tip & $35-38$ & 47 & $1.71 \pm 0.17$ & $1.35 \pm 0.19$ & 47 \\
\hline 0.2 mg/l IBA & 0.2 mg/l IBA & Node & $35-38$ & 47 & $1.43 \pm 0.18$ & $1.28 \pm 0.19$ & 47 \\
\hline \multirow{2}{*}{$\mathrm{MS}_{0}$} & $1.0 \mathrm{mg} / \mathrm{lBAP}+$ & Shoot tip & $38-40$ & 40 & $1.33 \pm 0.19$ & $1.33 \pm 0.22$ & 27 \\
\hline & $0.5 \mathrm{mg} / \mathrm{l} \mathrm{IBA}$ & Node & $38-40$ & 34 & $1.20 \pm 0.17$ & $1.20 \pm 0.22$ & 27 \\
\hline \multicolumn{2}{|c|}{$1.0 \mathrm{mg} / \mathrm{BAP}+1.0 \mathrm{mg} / \mathrm{l} \mathrm{BAP}+$} & Shoot tip & $32-35$ & 54 & $1.62 \pm 0.24$ & $1.87 \pm 0.22$ & 47 \\
\hline 0.5 mg/l IBA & $0.5 \mathrm{mg} / \mathrm{l} \mathrm{IBA}$ & Node & $32-35$ & 47 & $1.57 \pm 0.27$ & $1.57 \pm 0.29$ & 40 \\
\hline \multirow{2}{*}{$\mathrm{MS}_{0}$} & $2.0 \mathrm{mg} / \mathrm{BAP}+$ & Shoot tip & $38-40$ & 47 & $1.43 \pm 0.27$ & $1.36 \pm 0.19$ & 34 \\
\hline & 0.1 mg/l IBA & Node & $38-40$ & 40 & $1.34 \pm 0.19$ & $1.33 \pm 0.22$ & 34 \\
\hline \multicolumn{2}{|c|}{$2.0 \mathrm{mg} / \mathrm{BAP}+2.0 \mathrm{mg} / \mathrm{BAP}+$} & Shoot tip & $30-35$ & 67 & $1.60 \pm 0.20$ & $1.55 \pm 0.21$ & 54 \\
\hline 0.1 mg/l IBA & $0.1 \mathrm{mg} / \mathrm{l} \mathrm{IBA}$ & Node & $32-38$ & 60 & $1.56 \pm 0.22$ & $1.28 \pm 0.19$ & 47 \\
\hline \multirow{2}{*}{$\mathrm{MS}_{0}$} & $2.0 \mathrm{mg} / \mathrm{BAP}+$ & Shoot tip & $40-42$ & 40 & $1.34 \pm 0.19$ & $1.17 \pm 0.19$ & 27 \\
\hline & $0.2 \mathrm{mg} / \mathrm{l} \mathrm{IBA}$ & Node & $40-42$ & 34 & $1.20 \pm 0.18$ & $1.00 \pm 0.14$ & 27 \\
\hline \multicolumn{2}{|c|}{$2.0 \mathrm{mg} / \mathrm{BAP}+2.0 \mathrm{mg} / \mathrm{lBAP}+$} & Shoot tip & $32-38$ & 54 & $1.50 \pm 0.25$ & $1.25 \pm 0.18$ & 40 \\
\hline $0.2 \mathrm{mg} / \mathrm{l} \mathrm{IBA}$ & $0.2 \mathrm{mg} / \mathrm{l} \mathrm{IBA}$ & Node & $32-38$ & 54 & $1.37 \pm 0.17$ & $1.12 \pm 0.17$ & 34 \\
\hline \multirow{2}{*}{$\mathrm{MS}_{0}$} & $2.0 \mathrm{mg} / \mathrm{BAP}+$ & Shoot tip & $42-48$ & 27 & $1.25 \pm 0.21$ & $1.00 \pm 0.18$ & 20 \\
\hline & 0.5 mg/l IBA & Node & $42-48$ & 20 & $1.34 \pm 0.27$ & $1.00 \pm 0.24$ & 20 \\
\hline \multicolumn{2}{|c|}{$2.0 \mathrm{mg} / \mathrm{BAP}+2.0 \mathrm{mg} / \mathrm{lBAP}+$} & Shoot tip & $35-40$ & 47 & $1.57 \pm 0.27$ & $1.21 \pm 0.17$ & 27 \\
\hline
\end{tabular}




\begin{tabular}{lllllll}
$0.5 \mathrm{mg} / \mathrm{l}$ IBA & $0.5 \mathrm{mg} / \mathrm{l} \mathrm{IBA} \quad$ Node & $35-40$ & 34 & $1.40 \pm 0.22$ & $1.20 \pm 0.23$ & 27 \\
\hline
\end{tabular}

\section{Preservation of artificial seed}

To examine the viability of synthetic seed, seeds were kept in sterilized test tubes and stored them at $4^{\circ} \mathrm{C}$ and $0^{\circ} \mathrm{C}$ temperature in the refrigerator for $15,30,45,60,75,90$ and 120 days. To determine the viability of synthetic seeds after storage, seeds were brought out from refrigerator and cultured on $\mathrm{MS}_{0}$ medium (Table 4.

Table 4. Effect of preservation duration on the viability of synthetic seed (for each treatment 15 explants were used).

\begin{tabular}{ccc}
\hline \multirow{2}{*}{$\begin{array}{c}\text { Preservation duration } \\
\text { (days) }\end{array}$} & \multicolumn{2}{c}{ Germination rate after preservation } \\
\cline { 2 - 3 } 15 & At $4^{\circ} \mathrm{C}$ temperature (\%) & At $0^{\circ} \mathrm{C}$ temperature \\
\hline 30 & 100 & - \\
45 & 80 & - \\
60 & 67 & - \\
75 & 34 & - \\
90 & - & - \\
120 & - & - \\
\hline
\end{tabular}

After 15 days of storage at $4^{\circ} \mathrm{C}, 100 \%$ seeds were germinated on the culture media. After 30 days of storage $80 \%$ seeds and after 45 days of storage $67 \%$ seeds were germinated. But after 60 days of storage, the viability of seeds was decreased and only $34 \%$ seeds were germinated. When the seeds were cultured after 75,90 and 120 days of storage, no germination was observed. But in all cases the seeds stored at $0^{\circ} \mathrm{C}$ were failed to germinate. In this experiment it was observed that synthetic seed of Mimosa pudica L. can be stored at $4^{\circ} \mathrm{C}$ temperature for 60 days.

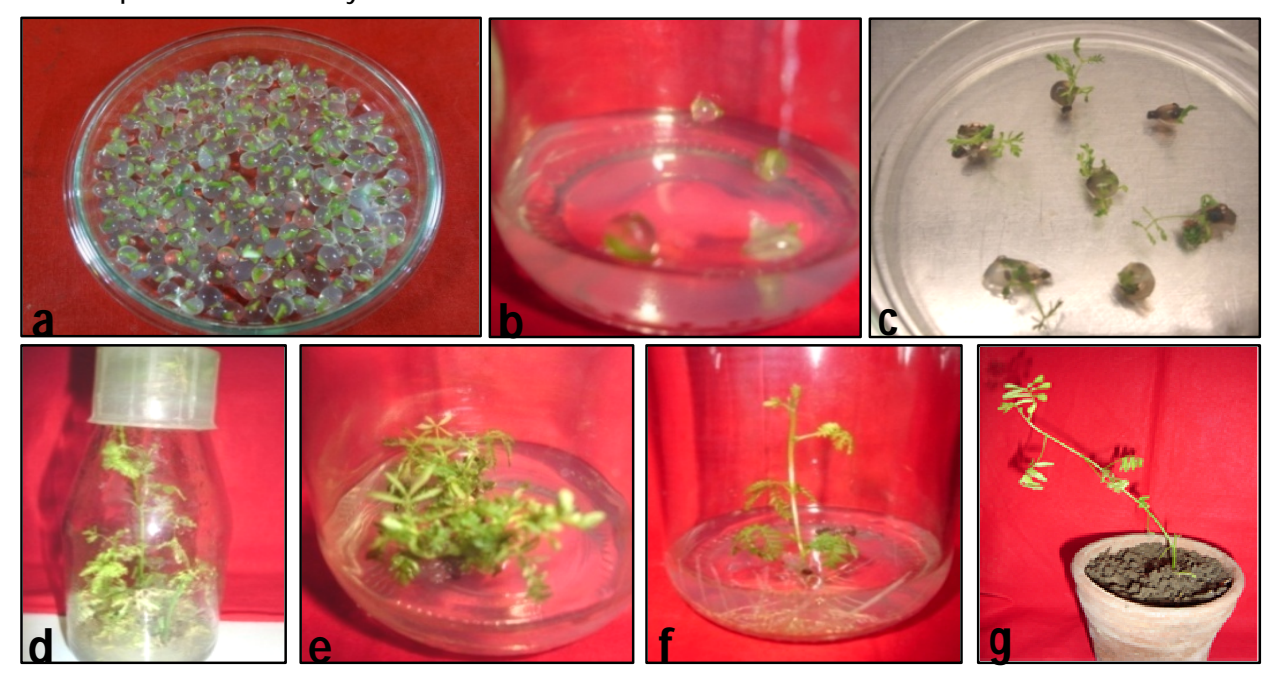

Fig. 1. Different stages of artificial seed germination. Artificial seeds encapsulated by sodium alginate (a)-(b), Germinated artificial seeds in $2.0 \mathrm{mg} / \mathrm{lBAP}$ (c), Artificial seed derived plant of shoot tip segments in $2.0 \mathrm{mg} / \mathrm{l} \mathrm{BAP}+0.2 \mathrm{mg} / \mathrm{l} \mathrm{NAA}$ (d), 
Artificial seed derived plant of nodal segments in $2.0 \mathrm{mg} / \mathrm{l} \mathrm{BAP}+0.2 \mathrm{mg} / \mathrm{lNAA}(\mathrm{e})$, Root induction from artificial seed germinated seeds, (f) and hardening of artificial seed derived plantlet in pot containing garden soil (g).

\section{Discussion}

Plant propagation using artificial or synthetic seeds developed from somatic and nonzygotic embryos opens up new vistas in agriculture. Artificial seeds make a promising technique for propagation of transgenic plants, non-seed producing plants, polyploids with elite traits and plant lines with problems in seed propagation. The technology of artificial seed was efficiently used for the cultivation of transgenic plant baikal skullcap (Scutellaria baicalensis Georgi) and common rue (Ruta graveolens L.) by encapsulating root fragment (Vdovitchenko and Kuzovkina, 2011). Encapsulation can be considered an important application of micropropagation, to improve the success of in vitro culture to synthetic seed technology. In the present investigation isolated shoot tip and nodal segments were encapsulated in $4 \%$ sodium alginate using in MS basal medium but Alatar and Faisal (2012) get maximum regeneration frequency (90.3\%) by using $3 \%$ sodium alginate in Lloyd and Mc Cown Woody Plant Medium (1981) for another medicinal plant Rauvolfia tetraphylla. Among the different concentrations and combinations of auxins and cytokinins, the highest $100 \%$ of shoot formation was observed in MS medium containing $2.0 \mathrm{mg} / \mathrm{l} \mathrm{BAP}+0.2 \mathrm{mg} / \mathrm{l} \mathrm{NAA}$ from shoot tip and nodal segments of Mimosa pudica $L$ whereas Awatef Badr-Elden (2013) reported maximum shoot multiplication response (61.60 \% shoots) in MS medium having $1.0 \mathrm{mg} / \mathrm{l}$ of BA, Same phytohormone was also found to produce best result in seed bead of mulberry (Machii 1992) and also was observed best in Withania somnifera (Siddique 2005) in artificial seed germination. It's suggested that combination treatment of auxin and cytokinin will be the best option to germinate artificial seed of M. pudica L.

Under the present investigation artificial seeds of Mimosa pudica L. were subjected to two storage temperature regimes at $4^{\circ} \mathrm{C}$ and $0^{\circ} \mathrm{C}$ and germination rate was examined. This investigation indicates that synthetic seed could be stored at $4^{\circ} \mathrm{C}$ for 60 days without loss of viability. Similar results were reported by Faisal et al. (2012) in another important medicinal plants Rauvolfia serpentina, maximum frequency of conversion into plantlets from encapsulated nodal segments stored at $4^{\circ} \mathrm{C}$ for 4 weeks was achieved on woody plant medium supplement with $1.0 \mathrm{mg} / \mathrm{BA}$ and $0.2 \mathrm{mg} / \mathrm{l} \mathrm{NAA}$. Ipekci and Gozukirmizi (2003) also observed that the encapsulated embryos of Paulownia elongate was survived when the synthetic seeds were stored at $4^{\circ} \mathrm{C}$ for 60 days and the germination rate was $32.40 \%$ and the result very perfectly supported our findings. This type of result was also supported the result of alfalfa seeds (Redenbaugh et al. 1987), Asparagus cooperi (Ghosh and Sen 1994), Eucalyptus citrisdora (Muralidharan and Mascarenhas 1995), Camellia (Janeiro et al. 1995), Mulberry (Machii 1992, Bapat et al. 1987). Kim and Park (2002) encapsulated calli obtained from a shoot tip of garlic, Allium sativum L., using a calcium alginate gel and stored for 40 days at $4^{\circ} \mathrm{C}$ and $88 \%$ of the encapsulated calli regenerated after 40 days of storage at $4^{\circ} \mathrm{C}$. Somatic embryo was used in synthetic seeds in egglplant and highest germination rate was $81 \%$ on MS +1.0 mg/1 BAP + 0.1 $\mathrm{mg} / 1 \mathrm{GA}_{3}$ and these encapsulated embryos did not loose germination even after storage of 45 days at $4^{\circ} \mathrm{C}$ (Huda and Bari 2007). Further, encapsulated somatic embryo retrieved from storage conditions was evaluated by Sudarshana et al. (2013) for its viability. The encapsulated embryos could be stored up to 4 months at $4^{\circ} \mathrm{C}, 20^{\circ} \mathrm{C}$ and $22^{\circ} \mathrm{C}$. Maximum conversion frequency of $90 \%$ was observed from encapsulated somatic embryos cultured on MS medium supplemented with $\mathrm{GA}_{3}(1.5 \mathrm{mg} / \mathrm{l})$, IAA $(0.5 \mathrm{mg} / \mathrm{l})$ and ascorbic acid $(40 \mathrm{mg} / \mathrm{l})$ and $22^{\circ} \mathrm{C}$ temperature they found to be optimum irrespective of storage periods.

\section{Conclusion}

The important medicinal plant Mimosa pudica L., in Bangladesh, belongs to the endangered and endemic category species. Thus it is important to propagate and conserve this plant species. Protocol establishment 
for artificial seed production, their germination and long term storage would provide promising avenues for the application of biotechnological tools towards its improvement and better utilization.

\section{References}

Alatar A, Faisal M. 2012. Encapsulation of Rauvolfia tetraphylla microshoots as artificial seeds and evaluation of genetic fidelity using RAPD and ISSR markers. J Med Plants Res 6, 1367-1374. http://dx.doi.org/10.5897/JMPR11.1632

Awatef M. Badr-Elden. 2013. An effective protocol for in vitro storage and ex vitro re-growth of strawberry capsules. Atlas Journal of Chemistry \& Biochemistry 1 (2): 30-38. http://dx.doi.org/10.5147/ajcb.2013.0081

Bapat VA, Mahatre M, Rao PS. 1987 Propagation of Morus indica L. (Mulbery) by encapsulated shoot buds. Plant Cell Rep. 6:393-395. http://dx.doi.org/10.1007/BF00269570

Faisal M, Alatar AA, Ahmad N, Anis M, Hegazy AK. 2012. Assessment of genetic fidelity in Rauvolfia serpentina plantlets grown from synthetic (encapsulated) seeds following in vitro storage at $4^{\circ} \mathrm{C}$. Molecules 17: 5050-5061. http://dx.doi.org/10.3390/molecules 17055050

Ghosh B, Sen S. 1994. Plant regeneration from alginate encapsulated somatic embryos of Asparagus cooperi Baker. Plant Cell Rep. 13: 381-385. http://dx.doi.org/10.1007/BF00234142

Gray D. 1987. Synthetic seed technology for the mass cloning of crop plants: Problem and perspectives. Hort Sci. 22: 795-814.

Hasan SMZ, Takagi H. 1995. Alginate coated nodal segments of Yam (Dioscorea spp) for germplasm exchange and distribution. Plant Genet Resour. Newslett.103: 32- 35.

Machii MH. 1992. In vitro growth of encapsulated adventitious bud in Mulberry. Morusalba L. Japan. J. Breed . 42: 553559. http:/ldx.doi.org/10.1270/jsbbs1951.42.553

Huda AKMN and Bari MA. 2007. Production of synthetic seed by encapsulating asexual embryo in eggplant (Solanum melongena L.). Int. J. Agr. Res. 2(9):832-837. http://dx.doi.org/10.3923/ijar.2007.832.837

Ipekci Z and Gozukirmizi N 2003. Direct somatic embryogenesis and synthetic seed production from Paulownia elongata. Plant Cell Rep. 22: 16-24. http://dx.doi.org/10.1007/s00299-003-0650-5

Janeiro LV, Ballester A and Vieitez AM. 1995. Effect of cold storage on somatic embryogenesis systems of Camelia. J. Hortic Sci. 70: $665-672$.

Kim YH and Janick J. 1987. Production of synthetic seeds of celery. Hort. Sci.22: p 89.

Kim MA and Park JK. 2002. High frequency plant regeneration of garlic (Allium sativum L.) calli immobilized in calcium alginate gel. Biotechnol. Bioprocess Eng. 7: 206-211. http://dx.doi.org/10.1007/BF02932971

Kim MA and Park JK. 2002. High Frequency Plant Regeneration of Garlic (Allium sativum L.) Calli Immobilized in Calcium Alginate Gel, Biotechnol. Bioprocess Eng. 7: 206-211. http://dx.doi.org/10.1007/BF02932971

Kinoshita I and Saito A. 1990. Propagation of Japanese white birch by encapsulated axillary buds. Regeneration of plantlets under aseptic conditions J. Jpn. For. Soc. 72: $166-170$.

Lloyd Gand Mc Cown B. 1981. Commercially-feasible micropropagation of mountain laurel, Kalmia latifolia, by use of shoot-tip culture. Comb. Proc. Intern. Plant Prop. Soc. 30: 421-427

Manda IPK, Bhattacharya A, Sood A, Ahuja PS. 2002. Propagation of tea (Camellia sinensis (L.) O. Kuntze) by shoot proliferation of alginate-encapsulated axillary bud stored at $4^{\circ} \mathrm{C}$. Curr. Sci. 83:941-944.

Maruyama E, Kinoshita I, Ishii K, Ohba K, Sakai A. 1996. Cryopreservation approach for the germplasm conservation of the tropical forest tree species: Cedrel aodorata L., Guazuma crinite Mart., and Jacaranda mimosaefolia D. Don. Plant Tissue Culture Letters 13: 297-310. http://dx.doi.org/10.5511/plantbiotechnology1984.13.297

Mathur J, Ahuja PS, Lal N, Mathur AK. 1989. Propagation of Valeriana wallichii DC. using encapsulated apical and axial shoot buds. Plant Sci. 60:111-116. http://dx.doi.org/10.1016/0168-9452(89)90050-2 
Muralidharan EM and Mascarenhos AF. 1995. Somatic embryogeneis Eucalyptus. In Somatic embryogenesis In: Woody plants, Mohan SJ, Pramod GK, Ronald JN (Ed), Kluwer Academic Publishers, The Netherlands 2:23-39.

Murashige T and Skoog F. 1962. A revised medium for rapid growth and bioassays with tobacco tissue cultures. Physiol. Plant. 15: 473 - 497. http://dx.doi.org/10.1111//.1399-3054.1962.tb08052.x

Murashige T. 1978. The Impact of plant Tissue culture on Agriculture. In: "frontirs of plant Tissue Culture". T.A. Thorpe (ed.) International Association for plant Tissue Cult., Colgary. 15-56 pp.

Pandey A, Chand S. 2005. Efficient plant regeneration from encapsulated somatic embryos of Hyoscyamus muticus L. Indian J. Biotec. 4:546-550.

Piccioni E and Standardi A. 1995. Encapsulation of micropropagated buds of six woody species. Plant Cell, Tissue Organ Cult. 42: 221-226. http://dx.doi.org/10.1007/BF00029990

Redenbaugh K, Slade D, Viss PR, Fujii J. 1988. Encapsulation of somatic embryos in synthetic seed coats. Hort. Sci. 22:803-809.

Siddique NA. 2005. Endangered medicinal plants in the Barind Tract and biotechnological approaches for their conservation. Ph. D. Thesis. Institute of Biological Sciences. Rajshahi University. Bangladesh.

Sudarshana MS, Rajashekar N, Niranjan MH. and Borzabad RK. 2013. In vitro regeneration of multiple shoots from encapsulated somatic embryos of Artemisia vulgaris L. IOSR-JPBS Vol 6, Issue 6, 11-15 pp.

Vdovitchenko M Yu. and Kuzovkinal N. 2011. Artificial seed preparation as the efficient method for storage and production of healthy cultured roots of medicinal plants. Rus J PI Phy, Vol. 58, No. 3, 524-530 pp. 